\title{
Modeling and Simulation Research of Ship-radiated Noise
}

\author{
Liu Jue ${ }^{1, a}$, Liu Pingxiang ${ }^{2, b}$, He Xudong ${ }^{1, c}$ \\ ${ }^{1}$ Science and Technology on Underwater Acoustic Antagonizing Laboratory, Shanghai, China, \\ ${ }^{2}$ Shanghai Marine Electronic Equipment Research Institute, Shanghai, China

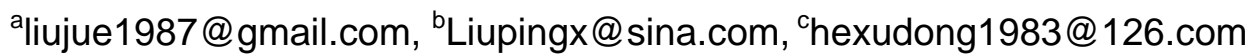

Keywords: Ship-radiated noise; Continuous spectrum variation curve; Line spectrum distribution

\begin{abstract}
The conventional method in target noise simulation which generally applied for verifying signal processing algorithm always use CW or LFM signal plus with pseudo-random sequences. But the reality target which has numerous machines complicates the radiated noise with propulsion, control, and habitability. Oceanic acoustics channel and target itself acoustic signature makes it more complex. This paper research on the characters of two basically different noise spectrum types which are continuous spectrum and line spectrum, and build two models mentioned above which based on target acoustic signature, sailing condition, and time-frequency statistical regularity. Simulation proved the validity of method which can apply to the hardware in the loop simulation, hardware system test, and underwater ordnance simulation.
\end{abstract}

\section{Introduction}

Actual ship-radiated noise has discrete distribution, the work of data acquisition need large cost and is generally time consuming. Hence it's unrealistically to depending on measured ship-radiated noise completely when researching on signal process, target classification and identification. Conventional ship-radiated noise simulating method is use CW or LFM signal plus with pseudo-random sequences, or building a continuous spectrum that have $-6 \mathrm{~dB}$ /octave attenuation characteristic plus with series of CW signal. Such mentioned method simulate the stable characteristic of ship-radiated noise, yet ship-radiated noise is also a random process which affected by oceanic acoustics channel (vary randomicity and periodicity) and target itself signature (speed, depth, tonnage, and propeller parameter, etc). This paper focus on the effect factor mentioned above, derive out empirical formula of continuous spectrum variation curve and build line spectrum statistic distribution model, therefore obtain equation of ship-radiated noise. Simulation analysis present in the last of the paper.

\section{Hydroacoustic characteristics of ship-radiated noise}

Urick [1] indicated that, the sources of noise on ships, submarines, and torpedoes can be grouped into the three major categories: machinery noise, propeller noise, and hydrodynamic noise. The spectral shape of these components consists of broadband noise named continuous spectrum and series of discrete frequency named line spectrum. Under normal circumstances, machinery noise and propeller noise are dominant noise source in the region of the spectrum.

Machinery noise results from pumps and motors used to keep onboard systems operating, which visualized as possessing a low-level continuous spectrum containing strong line components. Machinery noise is dominant noise source in low frequency end of the noise spectrum. Propeller noise results from the propulsion elements of a vessel, including the motor, shaft, and propeller, which can be subdivided into cavitation noise and blade-rate line components. Propeller cavitation noise caused by production and collapse of cavities which formed by the action of the propeller, has a continuous spectrum. Propeller cavitation noise usually dominates the high frequency end of the spectrum of ship noise, figure 1 shows diagrammatic cavitation noise spectrum. At low frequencies, the spectrum level of cavitation noise increases with frequency (this reverse slope tends to be obscured in measured data by other sources of noise). At high frequencies, its spectrum level 
decreases with frequency at the rate of about $6 \mathrm{~dB} /$ octave, or about $20 \mathrm{~dB} /$ decade. For ships and submarines, the peak frequency usually occurs within the frequency decade 100 to $1000 \mathrm{~Hz}$, and the peak frequency location shifts to lower frequencies at higher speeds and (in the case of submarines) at smaller depths. Propeller blade-rate spectrum caused by propeller beats and propeller blade interception, has a discrete spectral components. The frequency of the blade-rate series of line components is given by the formula: $f_{m}=m \cdot s \cdot n$, where $f_{m}$ is the frequency, in hertz, of the $m$ th harmonic of the blade-rate series of lines, $s$ is the number of blades on the propeller, and $n$ is the propeller rotation speed in number of turns per second. Propeller blade-rate line spectrum dominates 1- to $100-\mathrm{Hz}$ region of submarine spectrum, have commonly be used by sonar system for target estimation and identification.

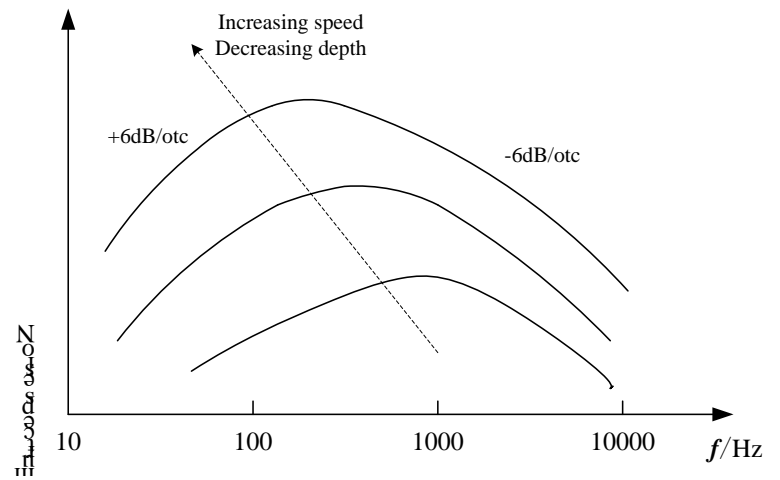

Fig.1 Variation of the spectrum of cavitation noise with speed and depth

\section{Modeling of ship-radiated noise}

For the dominant noise source of ship-radiated noise which consist of continuous spectrum and discrete line components are machinery noise and propeller noise, the mathematical model of ship-radiated noise can be formulated with,

$$
S(t)=[1+G(t)] \times S_{x}(t)+S_{l}(t) \text {. }
$$

where, $S(t)$ is ship-radiated noise, $G(t)$ is time domain waveform of modulation spectrum, $S_{x}(t)$ and $S_{l}(t)$ are time domain waveform of continuous spectrum and discrete line components. Following variability of continuous spectrum and statistic distribution regularity of line spectrum have been researched.

Variability of continuous spectrum. The characteristic of continuous spectrum can be described as 3 parameters that is the frequency of continuous spectrum peak symbolized as $f_{0}$, the corresponding spectrum level symbolized as $S L$ fo, and continuous spectrum variation curve $C(t)$. It is well known that target acoustic signature and sailing condition such as speed, depth, tonnage, diameter of propeller blade, hydrostatic pressure and so on can influence the shape of continuous spectrum waveform. Whereas Ross [2] give an empirical formula about peak frequency $f_{0}$ and target sailing condition,

$$
f_{0} \square \frac{1}{B} \sqrt{\frac{p_{0}}{\rho}} \sigma^{\frac{3}{2}} \text {. }
$$

where, $B$ is propeller blade width, $p_{0}$ is hydrostatic press, $\rho$ is density of sea water, $\sigma$ is cavitation number,

$$
\sigma=\frac{p-e}{\frac{\rho}{2}(\pi n D)^{2}} .
$$

where $U=\pi \cdot n \cdot D$ is linear speed of propeller blade, reference paper [3] give cavitation number calculation formula,

$$
\sigma=\frac{1.98}{v^{2}}(10+H) J_{P}^{2} .
$$


Assuming hydrostatic press $p_{0}$ and density of sea water $\rho$ are constant, formula [2] can be simplified to,

$$
f_{0} \square \frac{1}{D}\left(\frac{1.98}{v^{2}}(10+H) J_{P}^{2}\right)^{\frac{3}{2}} \text {. }
$$

where, $D$ is propeller blade diameter, $H$ is sailing depth, $v$ is speed, $J_{p}$ is advanced coefficient about propeller operation. For dominant noise source of continuous spectrum is propeller noise, and the frequency and spectrum level of continuous spectrum extremely influenced by propeller cavitation, it could give the following presumption. While target sailing speed under primary cavitation speed $V_{\mathrm{si}}$ which shows propeller cavitation has not yet started, $f_{0}$ and $S L_{f 0}$ are invariable. While target sailing speed is greater than saturated cavitation speed $V_{\text {SY }}$ which shows propeller noise is full cavitation, $f_{0}$ and $S L$ fo are also invariable. Then it could derive out the following empirical formula,

$$
\left\{\begin{array}{l}
f_{0}=\frac{A}{D}\left(\frac{1.98}{v^{2}}(10+H) J_{P}^{2}\right)^{\frac{3}{2}} \\
v_{S i}=2.7 \times \sqrt{\frac{10+H}{\left(1+\left(\frac{J_{P}}{\pi}\right)^{2}\right) \sigma_{i}}} \frac{J_{P}}{1-\omega} \\
v_{S Y}=2.82 \times J_{P} \sqrt{\frac{10+H}{1+\left(\frac{J_{P}}{\pi}\right)^{2}}} \\
\text { s.t. } \quad \min \left(\max \left(v, v_{S i}\right), v_{S Y}\right)
\end{array} .\right.
$$

where, $\omega$ propeller wake value, $A$ is a constant coefficient.

Continuous spectrum variation curve is take advantage of the results concluded in reference paper [3]. Assuming that,

-If target sailing speed is lesser than primary cavitation speed $V_{\text {si }}$ (i.e., $v \leqslant V_{\text {si }}$ ). At low frequencies (i.e., $f<f_{0}$ ), the spectrum level of continuous spectrum increases with frequency at the rate of about $3 \mathrm{~dB}$ /octave. At high frequencies (i.e., $f>f_{0}$ ), its spectrum level decreases with frequency at the rate of about $10 \mathrm{~dB}$ /octave.

-If target sailing speed is greater than saturated cavitation speed $V_{\mathrm{SY}}$ (i.e., $v \geqslant V_{\mathrm{SY}}$ ). At low frequencies (i.e., $f<f_{0}$ ), the spectrum level of continuous spectrum increases with frequency at the rate of about $6 \mathrm{~dB}$ /octave. At high frequencies (i.e., $f>f_{0}$ ), its spectrum level decreases with frequency at the rate of about $6 \mathrm{~dB}$ /octave.

-If target sailing speed is between primary cavitation speed $V_{\text {si }}$ and saturated cavitation speed $V_{\mathrm{SY}}$ (i.e., $V_{\mathrm{si}} \leqslant v \leqslant V_{\mathrm{SY}}$ ). It indicated that ship-radiated noise is in the moment of evolution between primary cavitation and saturated cavitation. At low frequencies (i.e., $f<f_{0}$ ), the spectrum level of continuous spectrum increases with frequency at the rate of about $3 q \mathrm{~dB}$ /octave, where $q$ is attenuation index of continuous spectrum variation curve. $q=1.2 N / N_{s i}-0.4$, where $N$ and $N_{s i}$ are the propeller primary cavitation and sailing rotation speed in number of turns per minute, $N / N_{s i}$ is value for evaluate propeller cavitation. At high frequencies, if $f_{0}<f<1000 \mathrm{~Hz}$, its spectrum level decreases with frequency at the rate of about $3 q \mathrm{~dB}$ /octave, if $f>1000 \mathrm{~Hz}$, its spectrum level decreases with frequency at the rate of about $2.1 \mathrm{~dB} /$ octave.

Continuous spectrum variation curve can be extracted form real noise data by linear phase low pass FIR filter as well. Such FIR filter presented in reference paper [4].

Spectrum level in the peak of continuous spectrum is related to propeller diameter $D$, propeller blade linear speed $U$, hydrostatic press $p_{0}$, peak frequency $f_{0}$, etc. This paper first working out spectrum level in the frequency of $5 \mathrm{kHz}$, then calculating the peak spectrum level $S L$ fo by the 
continuous spectrum variation curve described above. Empirical formula presented in reference paper [2] about spectrum level in the frequency of $5 \mathrm{kHz}$ is,

$$
\left\{\begin{array}{lr}
S L_{5 \mathrm{kHz}}=S L_{0}+40 \log (U / 10) & \text { (before cavitation) } \\
S L_{5 \mathrm{kHz}}=S L_{0}+40 \log (U / 10)+25+\delta S L & \text { (cavitation) } \\
S L_{0}=72.7+1.55 D-0.1 D^{2} & {[0.5 m \leq D \leq 6 m]} \\
\delta S L=13.75 N / N_{s i}-16.5 & {\left[1.2 \leq N / N_{s i} \leq 2\right]}
\end{array} .\right.
$$

Ship-radiated noise is a special classes of random process, which in addition has a certain power spectrum shape, but its amplitude can be considered a Gaussian distribution. Stochastic process theory indicate that, signal with Gaussian distribution getting through a linear system, the output signal having the same distribution. Thus, the emphasis of continuous spectrum simulation is constructing a filter which has similar amplitude frequency response to ship-radiated noise, then let Gaussian signal get through filter described above. Particular amplitude frequency response FIR adaptive filter [5,6] manage to achieve arbitrary structure of amplitude frequency and phase frequency response that will be well applied.

Statistic distribution regularity of line spectrum. Machinery noise and propeller noise is dominant line spectrum noise source. Line components of machinery noise produced by numbers of noise source which make up of respective fundamental frequencies and harmonic frequencies. Propeller line spectrum contains discrete spectrum blade rate components relevant to propeller rotation speed which also modulate continuous spectrum. Theoretical analysis from Tao Duchun [7], Wu Guoqing [8, 10], Shi Guangzhi [9] indicated that amplitude, waveform shape, and repetition period of modulation spectrum have regularity. Based on principle and effect of operation, line spectrum is classified into line spectrum of machinery noise, propeller blade rate line components, and modulation line spectrum created by propeller blade rate. There regularity will be analyzed carefully in the following paper.

1) Line spectrum of machinery noise: originates from repetitive vibration-producing process by pumps, fans, slot-pole, cylinders, etc. When many noise source are present, and environment factors such as vessel hull structure, sailing condition, and oceanic acoustics channel propagation, line spectrum of machinery noise is non stationary random process. Based on the nonstationarity of machinery line spectrum, there could assume factors such as number of noise source, respective fundamental frequency, number and distribution of respective harmonic frequency follow certain statistical rules.

$$
S_{\text {mac }}(t)=\sum_{\xi} \sum_{\zeta} a(\xi, \zeta)^{2} \exp (j 2 \pi f(\xi, \zeta) t) .
$$

where, $\xi$ is noise source number, assume it follow uniform distribution. $\zeta$ is harmonic frequency number corresponding to fundamental frequency, assume it follow rice distribution. $f(\xi$, $\zeta$ ) is frequency function restricted by fundamental and harmonic frequency which can be assume as obey continuous uniform distribution and chi-square distribution. $a(\xi, \zeta)$ is related amplitude, due to amplitude decrease with increasing harmonic multiply, assume it follow chi-square distribution.

2) Propeller blade rate line components: originates from water flow through or intercepted by the propeller blades. Its frequency related to the propeller rotation speed which used to sonar detection and recognition. Assume factors such as harmonic frequency number, harmonics distribution, and corresponding amplitudes follow certain statistical rules.

$$
S_{\text {prop }}(t)=\sum_{\zeta} a(\zeta)^{2} \exp (j 2 \pi f(\zeta) t)
$$

where, $\zeta$ is harmonic frequency number corresponding to blade rate line spectrum, propeller blade rate line spectrum normally has stable frequencies and large amount, it could assume harmonics number follow rayleigh distribution, the corresponding amplitude and multiply distribution follow chi-square distribution.

3) Modulation line spectrum: have the same origin with propeller blade rate line spectrum. 
Spectrum modulate continuous spectrum, the distribution rules similar to propeller blade rate line spectrum.

$$
S_{\bmod u}(t)=\sum_{\zeta} a(\zeta)^{2} \exp (j 2 \pi f(\zeta) t) .
$$

Because of modulation line spectrum stable and steady enough, it can assume harmonics number $\zeta$ and multiply harmonic distribution follow uniform distribution. And for integral multiple harmonic frequency have greater amplitude, its amplitude can be assumed to follow chi-square distribution while multiply additional coefficient in the frequency of integral multiple harmonic.

Finally, combined with continuous spectrum $S_{\text {conti }}(t)$, the ship-radiated noise can be formulated as,

$$
S(t)=\left[1+S_{\text {modu }}(t)\right] \times S_{\text {conti }}(t)+S_{\text {mac }}(t)+S_{\text {prop }}(t) \text {. }
$$

\section{Simulation}

Simulation based on a real foreign submarine parameters which is acquired from public reports. The basic parameters are: propeller diameter $D=3.1 \mathrm{~m}$, advanced coefficient $J_{p}=0.53$, propeller wake value $\omega=0.32$, propeller cavitation value when its primary occurred $\sigma_{i}=3.4$, propeller blade number $s=7$. Use noise data from USS-212 that is served in World War II as reference, it could calculate the coefficient $\mathrm{A}=0.23 \times 10^{4}$.

Continuous spectrum simulation. Assuming submarine sailing condition about speed and depth are $25 \mathrm{~m}$ and $7.5 \mathrm{kn}$, get these parameters into formula (6), it could resolve out primary cavitation speed $V_{\mathrm{si}}=6.66 \mathrm{kn}$, its blade linear speed and rotation speed are $U_{s i}=V_{\mathrm{si}} \times \pi / J_{p} \times(1-\omega) \approx 13.9 \mathrm{~m} / \mathrm{s}, N_{s i}=$ $U_{s i} \times 60 /(D \times \pi) \approx 85.7 \mathrm{r} / \mathrm{min}$, saturated cavitation speed $V_{\mathrm{SY}}=8.72 \mathrm{kn}$, peak frequency $f_{0}=151.0 \mathrm{~Hz}$.

Because of $V_{\mathrm{si}}<v<V_{\mathrm{SY}}$, submarine radiated noise is in the moment of evolution. Target current blade linear speed $U$ and rotation speed $N$ are $15.6 \mathrm{~m} / \mathrm{s}, 96 \mathrm{r} / \mathrm{min}$. It could obtain the continuous spectrum variation curve. When frequency $f>1 \mathrm{kHz}$, spectrum level decreases with frequency at the rate of about $2.1 \mathrm{~dB} /$ octave. When $150 \mathrm{~Hz}<f<1000 \mathrm{~Hz}$, attenuation index $q=1.2 \mathrm{~N} / N_{\mathrm{si}}-0.4 \approx 0.94$, spectrum level decreases with frequency at the rate of about $2.82 \mathrm{~dB} /$ octave. When $f<150 \mathrm{~Hz}$, spectrum level increases with frequency at the rate of about $2.82 \mathrm{~dB} /$ octave.

From formula (7), spectrum level in the frequency of $5 \mathrm{kHz}$ is calculated, $S L_{5 \mathrm{kHz}}=109.2 \mathrm{~dB}$. With continuous spectrum variation curve described above, spectrum level of continuous spectrum peak following solved, $S L_{150 \mathrm{~Hz}}=S L_{5 \mathrm{kHz}}+(10 \times 0.7) \times \log _{10}(5 / 1)+(10 \times 0.94) \times \log _{10}(1 / 0.15) \approx 121.54 \mathrm{~dB}$.

Considering frequency range from $0 \mathrm{~Hz}$ to $2500 \mathrm{~Hz}$ where target features are concentrate on. Figure 2 shows the continuous spectrum simulation result. Upper part of figure 2 shows design and expect amplitude frequency response of particular amplitude frequency response FIR adaptive filter, where the asterisk $(*)$ indicates expectation values which based on continuous spectrum curve, asterisk (-) indicates design amplitude frequency that approximate to expectation values. Results shows that filter reaches the predetermined expectation. Lower part of figure 2 shows spectrum result about Gaussian signal get through filter that is designed above. The result of continuous spectrum peak parameter is $158 \mathrm{~Hz} @ 121.4 \mathrm{~dB}$, which is close to the expected value. 

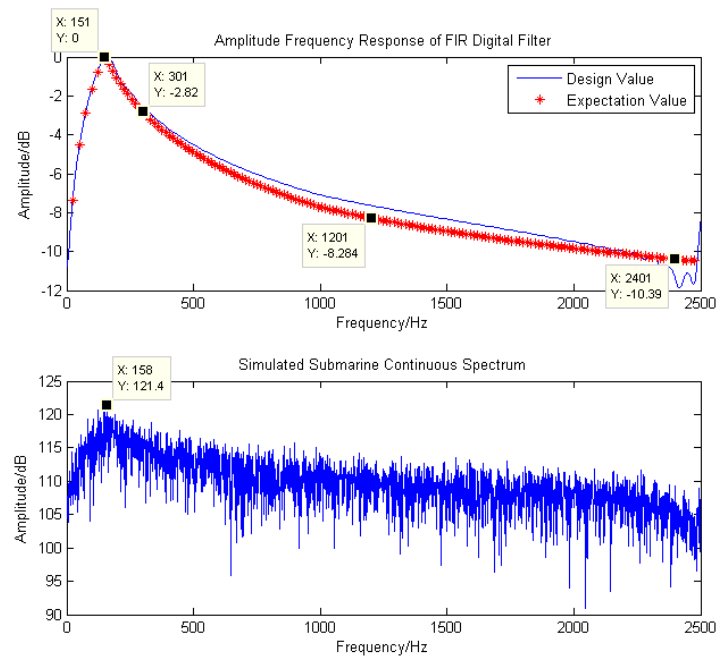

Fig.2 Simulation of ship-radiated noise continuous spectrum

Modulation spectrum and line spectrum simulation. In terms of statistic distribution regularity of line spectrum discussed above, simulation of modulation spectrum and line spectrum can be achieved. Table 1 present the concrete statistic distribution rule that parameters of machinery line spectrum, propeller blade rate line spectrum, and modulation line spectrum follows in a certain condition.

Table 1 Distribution regularity of line spectrum and modulation spectrum

\begin{tabular}{|c|c|c|c|c|c|}
\hline & $\begin{array}{c}\text { Noise source } \\
\text { number }\end{array}$ & $\begin{array}{l}\text { Fundamental } \\
\text { frequency }\end{array}$ & $\begin{array}{c}\text { Respective } \\
\text { harmonics } \\
\text { number }\end{array}$ & $\begin{array}{c}\text { Multiply harmonic } \\
\text { frequency } \\
\text { distribution }\end{array}$ & Amplitude \\
\hline $\begin{array}{c}\text { Machinery } \\
\text { line spectrum }\end{array}$ & $\begin{array}{c}\text { discrete } \\
\text { uniform } \\
\text { distribution } \\
(2 \sim 6)\end{array}$ & $\begin{array}{l}\text { continuous uniform } \\
\text { distribution } \\
(3 \sim 10 \mathrm{~Hz})\end{array}$ & $\begin{array}{l}\text { Rounding rice } \\
\text { distribution } \\
(3,5)\end{array}$ & $\begin{array}{c}\text { Rounding } \\
\text { noncentral } \\
\text { chi-square } \\
\text { distribution } \\
\lambda=32, v=6 \\
\end{array}$ & $\begin{array}{c}\text { Chi-spuare } \\
\text { distribution } \\
v=16\end{array}$ \\
\hline $\begin{array}{c}\text { Propeller } \\
\text { blade rate line } \\
\text { spectrum }\end{array}$ & & $\begin{array}{c}N \text { / } 60 \times 7 \mathrm{~Hz}, N \text { is } \\
\text { propeller blade } \\
\text { rotation speed, } \\
\text { r/min }\end{array}$ & $\begin{array}{c}\text { Rounding } \\
\text { rayleigh } \\
\text { distribution } \\
\sigma^{2}=8\end{array}$ & $\begin{array}{c}\text { Chi-spuare } \\
\text { distribution } \\
\quad v=8\end{array}$ & $\begin{array}{c}\text { Chi-spuare } \\
\text { distribution } \\
\quad v=2\end{array}$ \\
\hline $\begin{array}{l}\text { Modulation } \\
\text { line spectrum }\end{array}$ & & $N / 60 \mathrm{~Hz}$ & $\begin{array}{c}\text { discrete } \\
\text { uniform } \\
\text { distribution } \\
(8 \sim 16)\end{array}$ & $\begin{array}{l}\text { discrete uniform } \\
\text { distribution }(1 \sim 2) \\
\text { ×respective } \\
\text { harmonics number }\end{array}$ & $\begin{array}{c}\text { Chi-spuare } \\
\text { distribution } v=2 \\
\times 5 \delta(f-N / 60 \times k)+ \\
\text { Chi-spuare } \\
\text { distribution } v=2\end{array}$ \\
\hline
\end{tabular}

Table 2 Simulation results of line spectrum frequency and modulation frequency

\begin{tabular}{|c|l|}
\hline $\begin{array}{c}\text { Machinery line } \\
\text { spectrum (Hz) }\end{array}$ & $\begin{array}{l}497.14,529.57,556.59,561.99572 .68,691.68,707.89,721.03,740.31,758.12,829.02, \\
875.41,884.28,888.87,929.45,945.50,960.94,976.96,1105.09,1113.09,1149.57, \\
1153.13,1161.14,1217.20,1337.32\end{array}$ \\
\hline $\begin{array}{c}\text { Propeller blade rate } \\
\text { line spectrum (Hz) }\end{array}$ & $\begin{array}{l}22.35,55.89,67.06,78.24,89.42,100.60,111.78,122.95,134.13,145.31,156.49, \\
212.38\end{array}$ \\
\hline $\begin{array}{c}\text { Modulation line } \\
\text { spectrum (Hz) }\end{array}$ & $3.19,4.79,9.58,11.17,14.37,17.56,19.16,25.55,27.14$ \\
\hline
\end{tabular}




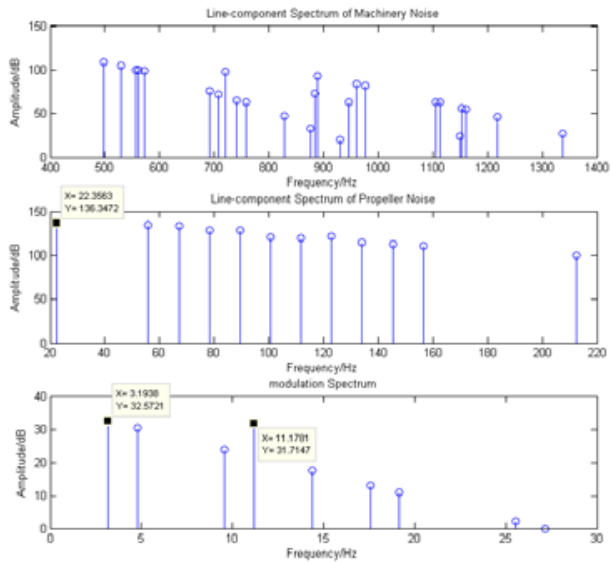

Fig.3 Amplitude frequency response characteristic of different line spectrums

Based on the distribution regularity presented on table 1 , simulation results of ship-radiated noise line components are display on figure 3 and table 2 about amplitude frequency distribution. Where amplitude and frequencies of line spectrum are time variation for the randomness of ship-radiated noise, and stable and steady frequency distribution based on propeller blades intercept water flow.

Figure 3 and table 2 shows that machinery noise line spectrum have 4 noise sources, which fundamental frequency are $5.40 \mathrm{~Hz}, 5.45 \mathrm{~Hz}, 6.80 \mathrm{~Hz}$, and $8.00 \mathrm{~Hz}$ respectively. Harmonics of those machinery line spectrum have wide distribution from about $400 \mathrm{~Hz}$ to $1400 \mathrm{~Hz}$. Fundamental frequency of propeller blade rate line spectrum is $s \times N / 60 \approx 11.17 \mathrm{~Hz}$, its harmonics mostly distribute under 20 multiply, which are under $200 \mathrm{~Hz}$ in frequency. Fundamental frequency of modulation line spectrum is $N / 60 \approx 1.59 \mathrm{~Hz}$, the amplitude of fundamental frequency and its harmonics decrease with the harmonics multiply increase in general, but at the frequency of $k \times S \times N$ / 60, amplitude will larger with respect to nearby harmonics. Modulation line spectrum mainly in the frequency under $30 \mathrm{~Hz}$.

Through the above simulation and analysis, it will obtain continuous spectrum and line spectrum of ship-radiated noise. The shape of continuous spectrum based on actual target signature and sailing condition such as speed, depth, tonnage, diameter of propeller blade, advanced coefficient, propeller wake value, and so on. Propeller cavitation which great influence the shape and spectrum level of continuous spectrum also be considered. Line spectrum shows both randomness and stability based on complexity of machinery noise, environment effect such as hull and oceanic acoustic channel, propeller blades vibration, rotation, and intercept water flow. wherein, the former two affect line spectrum randomness. Thus, according to the formula (11), ship-radiated noise is simulated, and power spectrum in the frequency of $0 \sim 2500 \mathrm{~Hz}$ where target features are concentrate on is shown in figure 4.

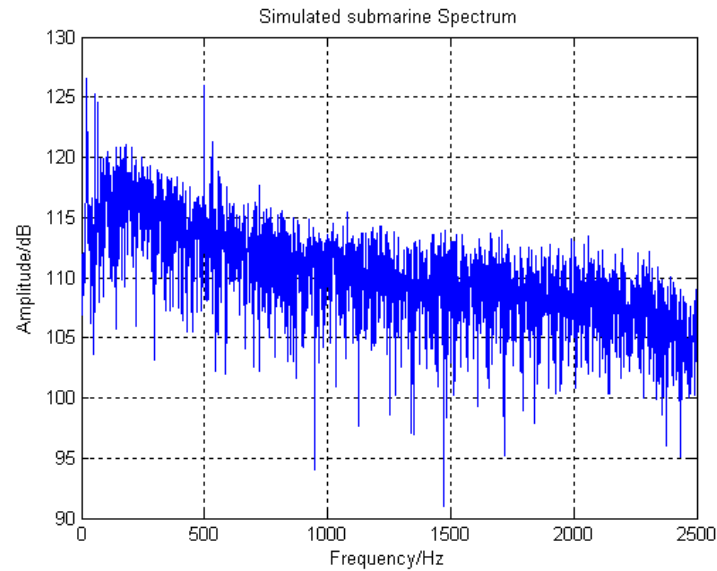

Fig.4 Spectrum of ship-radiated noise simulation $(0 \sim 2500 \mathrm{~Hz})$

\section{Summary}


Conventional ship-radiated noise simulating method is use CW or LFM signal plus with pseudo-random sequences, or building a continuous spectrum that have $-6 \mathrm{~dB}$ /octave attenuation characteristic plus with series of CW signal. But the reality target which has numerous machines complicates the radiated noise with propulsion, control, and habitability. Oceanic acoustics channel and target itself acoustic signature makes it more complex. This paper research on the randomness and stability of ship-radiated noise, building the continuous spectrum and line spectrum simulation model. Continuous spectrum simulation model is based on actual target signature and sailing condition such as speed, depth, tonnage, diameter of propeller blade, advanced coefficient, propeller wake value, etc. Propeller cavitation which great influence the shape and spectrum level of continuous spectrum also be considered. Line spectrum simulation model is based on environment interaction and operation principle of machinery line spectrum, propeller blade rate line components, and modulation line spectrum which have statistic distribution regularity. Simulation about a real submarine radiated noise in a certain sailing condition where parameters are acquired from public reports is discussed. Results shows that ship-radiated noise simulation model can be used to simulate target with different acoustic signature and sailing condition. The simulation model can apply to the hardware in the loop simulation, hardware system test, and underwater ordnance simulation.

\section{References}

[1] Robert J. Urick: Principles of underwater sound, edited by Diane Heiberg and Janet Davis, New York, 1981

[2] Donald Ross: Mechanics of Underwater Noise, Pergamon Press, New York, 1976

[3] Qian Xiaonan: Propeller Noise of Vessel, Shanghai Jiaotong University Press, Shanghai, 2011

[4] Hu Guangshu: Digital signal Processing, tsinghua University Press, Beijing, 2003

[5] Yan Shengfeng, Ma Yuanliang: Design of FIR filter with specific frequency response in underwater acoustic signal processing, Acoustic Application, 2003, 22(2): 30-34

[6] Yang Yixin, Sun Chao: On Adaptively Obtaining Optimum Cost Function in Designing FIR (Finite Impulse Response) Digital Filters, Jounal of Northwest Polytechnical University, 2002, 20(4): 554-557

[7] Tao Duchun: A Study on Ship Radiated Noise Rhythms(I)——Mathematical Model and Power Spectrum Density, Acta Acustica, 1983, 8(2):65-76

[8] Wu Guoqing: Two ways of propeller recognition feature extrsction, Acta Acustica, 1993, 18(3):210-216

[9] Shi Guangzhi, Hu Junchuan: Theoretical analysis of the structure law of ship radiated-noise demodulation spectrum harmonic clan feature, Acta Acustica, 2007, 32(1):19-25

[10]Wu Guoqing: Ship radiated-noise recognition (I) the overall framework, analysis and extraction of line-spectrum, Acta Acustica, 1998, 23(5):394-400 